\title{
Besondere Sitzung vom 25. April 1914.
}

Vorsitzender: Hr. W. Will.

Der Yorsitzende begrüßt als Gäste die Vertreter des Ministeriums für Handel und Gewerbe, der Tecbnischen Deputation für Gewerbe, des Kaiserlichen Patentamts, des Vereins zur Wahrung der Interessen der Chemischen Industrie Deutscblands, sowie des Vereins zur Beförderung des Gewerbfleißes, ferner die auswärtigen Mitglieder, HHrn.: J. v. Braun (Breslau), L. Gans (Fraukfurt a. M.), R. Hagenbach (Höchst), R. Meyer (Braunschweig), F. Weigert (Leipzig), A. Hantzsch (Leipzig), E. Wedekind (Straßburg), P. Duden (Höchst).

Hierauf erhält der Reduer des Abends, Hr. R. Willstätter, das Wort zu seinem Vortrage über

\section{\Pilanzenfarbstoffe«.}

An den mit lebhaftem Beifall aufgenommenen Vortrag schließt der Vorsitzende die folgende Ansprache:

-Wir sind gewöhnt, an den Abenden, die den zusammenfassenden Vorträgen hervorragender Fachgenossen gewidmet sind, von ibnen auf hochragende Aussichtspunkte geführt zu werden, von welchen ein freier Überblick gewonnen wird über ein umfassendes Gebiet unserer wissenschaftlichen Arbeit, um so von boher Warte aus eine vollständigere und richtigere Würdigung des in unserer Arbeit erworbenen geistigen Besitzes zu erbalten. Von dem heutigen Abend bat man ron vornherein angenommen, daß er ein wertvoller und genußreicher für uns werden würde, wie die glänzende Versammlung von Fachgenossen erweist, die wir hier um uns sehen. Die Kundgebung, mit welcher Sie den Herrn Vortragenden beglückwünscht haben, läßt keinen Zweifel, wie hoch diesen Erwartungen entsprochen worden ist. Freilich war der Gegenstand, über den wir unterhalten werden sollten, von nicht gewöhnlichem Interesse. Seit der Zeit, in der Wöhler den Harnstoff herzustellen gelehrt hat, sind wir ein gutes Stück vorwärtsgekommen in der chemischen Forschung, die sich mit den Produkten des Lebens der Tier- und Pflanzenwelt beschäftigt. Seitdem wir gelernt baben, das Alizarin, den Indigo, die Zucker, die Eiweißkörper, 
den Kautschuk und andere derartige Stoffe in unseren Laboratorien darzustellen, betrachten wir es beinahe ais etwas Selbstverständliches, $\mathrm{da} B$ all die chemischen Produkte des organischen Lebens unserer Erkenntnis zugänglich sind und in unsern Arbeitswerkstätten so gut und besser wie in dem Zellenlaboratorium der Natur bergestellt werden können. Und doch ging es wie eine Offenbarung durch die Gemeinde der Chemiker, als die Kunde $\mathrm{kam}$, daß man das Chlorophyll isoliert, seine chemische Zusammensetzung bestimmt und in ibr solch merkwürdige Aufklärung für die Funktion dieser Substanz gefunden babe. Wie früher für den Blutfarbstoff im tierischen Körper, ist jetzt ein überraschender Einblick in die physiologische Wirkung des Farbstoffs gewonnen, der die Energie der Sonnenstrablen aufzusammeln und mit ihr aus Kohlensüure, Wasser und einigen wenigen anderen Stoffen einfachster Art in der Pflanzenzelle all die Wunder bervorzubringen vermag, die uns in der aufkeimenden Pllanzenwelt des jungen Früblings draußen entzücken.

* Aber wie reich hat der Vortragende diese schöneu Resultate durch seine veuerev Arbeiten verrollkommnet, deren Erfolg einen so umfassenden Einblick in die Farbenschöpfungen der Blütenwelt bietet, welche die Natur in unseren Feldern und Gärten hervorzaubert. Man weiß nicht, soll man mehr die Feinheit und sorgfältige Durcharbeitung der Methoden, den Reichtum der neuen Erkenntnis oder die so überzeugende Klarheit und Schönbeit der Schilderung bewundern.

- Die Versammlung hat ibren Dank scbon in nicht mißzuverstehender Weise kundgegeben. Ich habe die Aufgabe, ihm einige Worte zu leiben, und wenn ich, verehrter Herr Kollege, Ihnen daher nochmals unseren herzlichsten Dank ausspreche, so darf ich hinzufügen, daß es mir eine besondere Freude war, noch einmal am Ende meiner Amtszeit den Vorsitz bei einem solchen Vortrag fülren zu dürfen, der sicherlich in unser aller Erinnerung haften wird als ejn Markstein in der Geschichte unserer Wissenschalt.k

Der Vorsitzende:

W. Will.
Der Schriftführer:

A. Bannow. 